\section{World Health Organization Regional Office for Africa Weekly Bulletin on Outbreaks and Other Emergencies}

\author{
Benido Impouma, Brett N. Archer, \\ Okot Charles Lukoya, Esther L. Hamblion, \\ Ibrahima Socé Fall
}

Author affiliation: World Health Organization Regional Office for Africa, Cité du Djoué, Democratic Republic of the Congo

\section{DOI: https://doi.org/10.3201/eid2407.180573}

$\mathrm{T}$ he basis for effective public health action is accurate and timely information (1). During emergencies, a fundamental public health tool is rapid, proactive, and transparent communication. Information gathered and disseminated during emergencies not only guides public health authorities but also encourages communities to adopt protective behaviors, triggers a heightened level of disease surveillance across borders, and reduces confusion among national authorities and communities (2). Although the International Health Regulations (2005) have provided a strong system for urgent communications (3), these alerts often remain siloed among a few parties. For several decades, lack of information sharing or incompatible communication systems have remained paralyzing factors for complex emergency responses (4). Indeed, among the lessons from the 2014-2016 Ebola virus disease epidemic was the value of effective communication and transparency in reporting (1).

The Transformation Agenda of the World Health Organization (WHO) Secretariat in the African Region 2015-2020 has 4 major objectives, 1 of which is improved strategic and effective communication (5). In keeping with this objective, in March 2017, the WHO Regional Office for Africa launched the Weekly Bulletin on Outbreaks and Other Emergencies (the Bulletin). The Bulletin (Figure) is not intended to compete with or replace the more traditional communication strategies, which range from targeted risk communications and media to peer-reviewed scientific publications. Rather, the Bulletin aims to bridge the gaps within this spectrum, providing real-time actionable updates on the status of new and ongoing events, while highlighting actions taken and gaps that need addressing by Member States and partners.

However, providing regular communications on emergencies in the African region is no easy task. The sheer scope and magnitude of emergencies presents an enormous challenge. Capacity on the ground is often limited, and

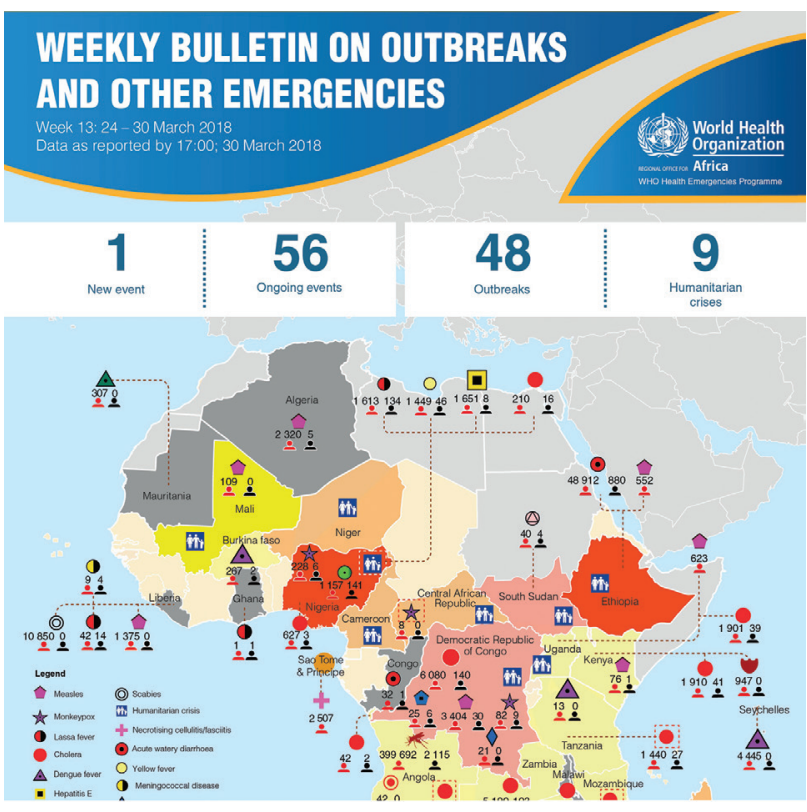

Figure. Cover of recent edition of the Weekly Bulletin on Outbreaks and Other Emergencies, published by the World Health Organization Regional Office for Africa.

investigations and response efforts often take precedence over information dissemination. Communications must also proceed amid a great deal of uncertainty; events are often rapidly evolving or subjected to political sensitivities. For too long, these challenges have precluded timely communications about emergencies in the African region; information often remains unpublished, is published in retrospect, or is credited to authors outside the region.

The Bulletin provides a platform for overcoming these challenges. Through participation in the writing process, it provides a mechanism for WHO country offices to rapidly communicate updates to a wide audience. In 2017, the Bulletin published 43 editions, including 245 articles, and disseminated them directly to a growing readership of $\approx 2,000$ members and posted them on social media and public health information websites, including but not limited to ProMED-mail, Outbreak News Today, and ACAPS (Assessment Capacities Project).

With the support of WHO Member States, it is our hope that the Bulletin will continue to play a major role in improving communication in the region. We welcome readers to join our mailing list.

\section{Acknowledgments}

The regular publication of the Bulletin would not be possible without the open contributions of WHO staff across the African Region; generous sharing of information by ministries of health and partners; and a team of writers, editors, graphics designers, and other staff in the WHO Health Emergencies Programme. 


\section{About the Author}

Dr. Impouma is program manager of Health Emergency Information \& Risk Assessment for the WHO Health Emergencies Programme within the Regional Office for Africa. His primary research interests include prevention and control of infectious disease outbreaks, global public health, monitoring and evaluation, and health information systems.

\section{References}

1. World Health Organization. WHO leadership statement on the Ebola response and WHO reforms [cited 2017 Feb 15]. http://www.who.int/csr/disease/ebola/joint-statement-ebola/en/

2. O’Malley P, Rainford J, Thompson A. Transparency during public health emergencies: from rhetoric to reality. Bull World Health
Organ. 2009;87:614-8. http://dx.doi.org/10.2471/BLT.08.056689

3. World Health Organization. International Health Regulations (2005). Geneva: The Organization; 2008.

4. Burkle FM. Lessons learnt and future expectations of complex emergencies. BMJ. 1999;319:422-6. http://dx.doi.org/10.1136/ bmj.319.7207.422

5. World Health Organization Regional Office for Africa. The Transformation Agenda of the World Health Organization Secretariat in the African Region 2015-2020 [cited 2017 Feb 15]. http://who.insomnation.com/sites/default/files/ pdf/9789290232827.pdf

Address for correspondence: Benido Impouma, WHO Regional Office for Africa, WHO Health Emergencies Programme, Health Emergency Information \& Risk Assessment, PO Box 06, Cité du Djoué, Brazzaville, Congo; email: impoumab@who.int

\section{April 2017: Emerging Viruses}

- Biologic Evidence Required for Zika Disease Enhancements by Dengue Antibodies

- Neurologic Complications of Influenza B Virus Infection in Adults, Romania

- Implementation and Initial Analysis of a Laboratory-Based Weekly Biosurveillance System, Provence-Alpes-Côte d'Azur, France

- Transmission of Hepatitis A Virus through Combined Liver-Small Intestine-Pancreas Transplantation

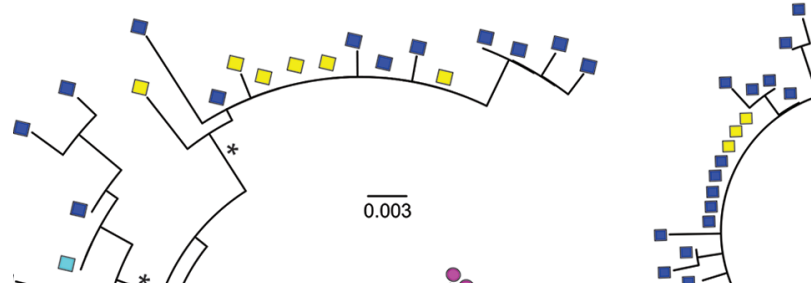

- Influence of Referral Pathway on Ebola Virus Disease Case-Fatality Rate and Effect of Survival Selection Bias

- Plasmodium malariae Prevalence and csp Gene Diversity, Kenya, 2014 and 2015

- Presence and Persistence of Zika Virus RNA in Semen, United Kingdom, 2016

- Three Divergent Subpopulations of the Malaria Parasite Plasmodium knowlesi

- Variation in Aedes aegypti Mosquito Competence for Zika Virus Transmission

- Outbreaks among Wild Birds and Domestic Poultry Caused by Reassorted Influenza A(H5N8) Clade 2.3.4.4 Viruses, Germany, 2016

- Highly Pathogenic Avian Influenza A(H5N8) Virus in Wild Migratory Birds, Qinghai Lake, China
- Design Strategies for Efficient Arbovirus Surveillance

- Typhus Group Rickettsiosis, Texas, 2003-2013

- Detection and Molecular Characterization of Zoonotic Poxviruses Circulating in the Amazon Region of Colombia, 2014

- Reassortment of Influenza A Viruses in Wild Birds in Alaska before H5 Clade 2.3.4.4 Outbreaks

- Incidence and Characteristics of Scarlet Fever, South Korea, 2008-2015

- Markers of Disease Severity in Patients with Spanish Influenza in the Japanese Armed Forces, 1919-1920

- Molecular Identification of Spirometra erinaceieuropaei in Cases of Human Sparganosis, Hong Kong

- Zika Virus Seroprevalence, French Polynesia, 2014-2015

- Persistent Arthralgia Associated with Chikungunya Virus Outbreak, US Virgin Islands, December 2014February 2016

- Assessing Sensitivity and Specificity of Surveillance Case Definitions for Zika Virus Disease

- Detection of Zika Virus in Desiccated Mosquitoes by Real-Time Reverse Transcription PCR and Plaque Assay

- Surveillance and Testing for Middle East Respiratory Syndrome Coronavirus, Saudi Arabia, April 2015-February 2016

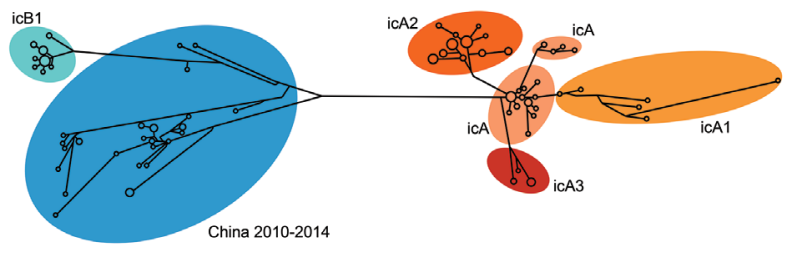

